Onkologe 2009 $\cdot 15: 84$

DOI 10.1007/s00761-008-1567-y

(c) Springer Medizin Verlag 2009

K. Höffken ${ }^{1}$, M. Bamberg' ${ }^{2}$, P.M. Schlag ${ }^{3}$

${ }^{1}$ Klinik für Innere Medizin II, Universitätsklinikum Jena

${ }^{2}$ Radiologische Universitätsklinik, Abt. für Strahlentherapie, Tübingen

${ }^{3}$ Charité Comprehensive Cancer Center, Charité - Campus Mitte, Berlin

\title{
Warum tun wir nicht, was wir wissen?
}

sorgung von Krebspatienten alles berücksichtigt, was den aktuellen Kenntnisstand der Medizin beinhaltet, dass wir also noch nicht alles tun, was wir wissen.

Aus diesem Grund beginnen wir mit dem vorliegenden Heft eine Serie zur komplementären Onkologie, die eingeleitet wird durch den Beitrag von Heimpel und Mitautoren zu „wissenschaftlich begründeten Verfahren und Methoden mit umstrittener Wirksamkeit“. Jedem der Autoren kann bescheinigt werden, dass er sich mit großer Erfahrung in der Onkologie und großer Objektivität seit Jahren diesem schwierigen Thema eines Bereiches der Medizin widmet, in dem Schnittmengen ungenutzt existieren.

Mit diesem Beitrag wird der Boden bereitet für die folgenden Beiträge, die in den nächsten Heften folgen und die komplementäre Onkologie unter wissenschaftlichen Gesichtspunkten abhandeln. Sie stammen aus der Feder von Jutta Hübner und von ihr gebetenen Mitautoren und werden die jeweils bekannten Fakten aus Grundlagenforschung, tierexperimenteller Forschung, klinischen Studien darlegen und mit einer rationalen Empfehlung enden.

Mit diesem Wissen kann der praktisch tätige Onkologe seine Patienten beraten und eventuell auch sein Repertoire der therapeutischen Optionen erweitern.
Wir, die Herausgeber von „Der Onkologe“, sind überzeugt, dass dadurch ein positiver Beitrag zur Versorgung von Krebspatienten geleistet wird.

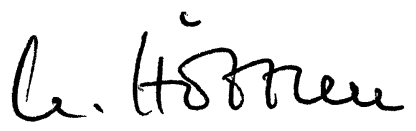

Prof. Dr. K. Höffken
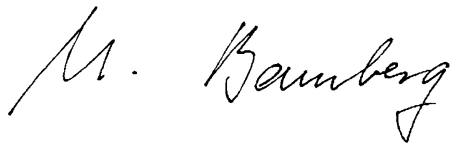

Prof. Dr. M. Bamberg

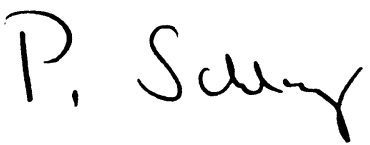

Prof. Dr. Dr. h.c. P.M. Schlag

\section{Korrespondenzadresse Prof. Dr. K. Höffken}

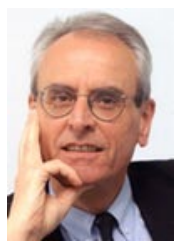

Universitätsklinikum Jena Klinik für Innere Medizin II Erlanger Allee 101 07747 Jena hoeffken@med.uni-jena.de 